\title{
Analysis of co-factor function in a glucocorticoid-resistant small cell carcinoma cell line
}

\author{
C E Waters ${ }^{1}$, A Stevens, A White and D W Ray \\ Endocrine Sciences Research Group, 3.810 Stopford Building, Schools of Biological Sciences and Medicine, University of Manchester, Oxford Road, \\ Manchester M13 9PT, UK \\ ${ }^{1}$ Centre for Vascular Biology and Medicine, Guy's Campus, King's College London, University of London, London SE1 9UL, UK \\ (Requests for offprints should be addressed to DW Ray and A White; Email: David.W.Ray@man.ac.uk or awhite@man.ac.uk)
}

\begin{abstract}
Human small cell lung carcinoma (SCLC) tumours exhibit neuroendocrine differentiation, secreting hormones such as ACTH and related peptides. While glucocorticoids inhibit ACTH secretion from the pituitary, this does not occur in SCLC tumours and SCLC cell lines. Failure of glucocorticoids to suppress ACTH peptides is accompanied by a global lack of glucocorticoid action in a number of SCLC cell lines.

In the human SCLC cell line, COR L103, activation of a human tyrosine aminotransferase (TAT3)-luciferase reporter gene is resistant to glucocorticoids despite similar glucocorticoid receptor (GR) expression to the glucocorticoid-sensitive A549 human lung cancer cell line; moreover, the GR is free of deleterious mutations. Overexpression of a wild-type GR restores glucocorticoid regulation of TAT3-luciferase, and this is enhanced when the activation function (AF)-2 domain is deleted but much
\end{abstract}

reduced when the AF-1 domain is deleted. This suggests aberrant AF-2 activation domain function. We identified defective steroid receptor co-activator 1 (SRC1) recruitment to the GR AF-2 in COR L103 cells, although SRC1 was successfully recruited to the steroid $X$ receptor with rifampicin, suggesting a defect in the GR. Analysis of other GR C-terminal co-factors identified increased expression of nuclear co-repressor (NCoR) in COR L103 cells. To determine the impact of this, NCoR was over-expressed in A549 cells, where it reduced GR transactivation by $55 \%$.

In summary, glucocorticoid resistance is associated with altered SRC protein recruitment and increased expression of $\mathrm{NCoR}$ in these SCLC cells, suggesting that glucocorticoid sensitivity may be modified by subtle changes in co-factor recruitment.

Journal of Endocrinology (2004) 183, 375-383

\section{Introduction}

The ectopic adrenocorticotrophin (ACTH) syndrome is a dramatic clinical example of glucocorticoid resistance where secretion of ACTH peptides is markedly resistant to the normal negative feedback of circulating glucocorticoids. This property is so common that it is exploited in a clinical diagnostic test (Nieman 2001). The most frequent type of tumour giving rise to the ectopic ACTH syndrome is small cell lung cancer (SCLC), with up to $50 \%$ of SCLC patients having biochemical or clinical features of aberrant ACTH production, although usually other clinical features predominate at diagnosis (Odell 1991). SCLC makes up $20 \%$ of all human lung cancers, and has a very poor prognosis, with widespread early metastases. SCLC is thought to result from malignant transformation of a primitive progenitor cell that then undergoes a pattern of dysdifferentiation (Baylin \& Mendelsohn 1980).

We have previously studied a panel of human SCLC lines and found them to be markedly glucocorticoid resistant. These cell lines produce ACTH-related peptides, and this production is resistant to dexamethasone inhibition (Stewart et al. 1989, White et al. 1989, Clark et al. 1990). In addition, transfection of glucocorticoid reporter (GR) genes, either derived from the mouse mammary tumour virus long terminal repeat (MMTV), or a simple synthetic reporter gene with three copies of the glucocorticoid responsive element of the human tyrosine aminotransferase (TAT3) gene are not activated either by natural or synthetic glucocorticoids (Ray et al. 1994, Huynh et al. 2002).

In most of the SCLC cell lines, the cause of resistance has been identified as a lack of expression of full-length GR, either due to a splicing defect, GR $\Delta$ (Gaitan et al. 1995) and GR $\gamma$ (Ray et al. 1996, Rivers et al. 1999), or a decrease in gene expression (Ray et al. 1994, 1996). However, one of the cell lines, COR L103, showed an unexpected pattern of resistance. Whilst over-expression of wild-type GR is sufficient to confer glucocorticoid regulation on both the MMTV and the TAT3 reporter 
genes in most of the cell lines, over-expression of wildtype GR only confers glucocorticoid regulation onto the TAT3 reporter in COR L103 cells (Huynh et al. 2002). Therefore, even in the presence of wild-type GR $\alpha$, the MMTV template is not regulated by glucocorticoids in COR L103 cells. As transactivation of MMTV requires both the $\mathrm{N}$ - and C-terminal transactivation domains of the GR to interact, but either domain acting alone can transactivate TAT3, this suggests aberrant function of one of the two major GR transactivation domains (IniguezLluhi et al. 1997).

The aim of these studies, therefore, was to test the hypothesis that dysfunction in co-factor recruitment to the GR may underlie glucocorticoid resistance in the COR L103 cell line, defining a novel mechanism which may have implications for SCLC proliferation and differentiation.

\section{Materials and Methods}

\section{Cell culture}

COR L103 SCLC cells, derived from a patient with histologically confirmed bronchiogenic SCLC were generously donated by Professor Peter Twentyman, MRC, Cambridge, UK (White et al. 1989). Cells were cultured in RPMI 1640 medium supplemented with $2.5 \%$ fetal calf serum (FCS), $1 \mathrm{mM}$ sodium pyruvate, $10 \mathrm{mM}$ HEPES, $10 \mu \mathrm{M}$ human transferrin, $5 \mu \mathrm{g} / \mathrm{ml}$ bovine insulin, $3 \times 10^{-8} \mathrm{M}$ sodium selenite, $4 \mathrm{mM}$ L-glutamine. COS 7 and A549 cells, purchased from the European collection of animal cell cultures (ECACC), were cultured in Dulbecco's modified Eagles' medium (DMEM) with Glutamax (Gibco BRL, Paisley, Strathclyde, UK) supplemented with 10\% FCS. Dexamethasone was purchased from Sigma Chemicals (Poole, Dorset, UK).

\section{Plasmids}

Wild-type rat GR expression vector (p6 RGR), along with the GR activation function (AF) deleted constructs

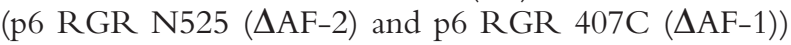
and the promoter construct TAT3-luciferase (luc) were a kind gift from Dr J Iniguez-Lluhi (University of California San Francisco) (Iniguez-Lluhi et al. 1997). The steroid receptor co-activator 1 (SRC1) receptor interacting domain (residues 570-780) and the human GR Cterminus (residues 526-777) were inserted into the pACT and pBIND vectors (components of the Checkmate Mammalian two-hybrid system (Promega, Southampton, Hants, UK)) respectively, as previously described (Stevens et al. 2003a) to give pBIND-SRC1 and pACT-GR. VP16/SXR, consisting of the steroid $\mathrm{X}$ receptor (SXR) ligand binding domain fused to the activation domain of VP16, was a kind gift from Professor $\mathrm{K}$ Chatterjee
(University of Cambridge, Cambridge, Cambs, UK) (Wentworth et al. 2000). pcDNA3-NCoR, an expression vector for human nuclear co-repressor (NCoR) was kindly donated by Johnson Liu (National Institute of Health, Washington DC, USA). The GAL4 responsive reporter gene used in mammalian two-hybrid studies, pG5-luc, was from Promega (Stevens et al. 2003a). CMV- $\beta$ Gal was described previously (Ray et al. 1999). CMV-renilla was from Promega (Stevens et al. 2003b).

\section{Transfection}

COR L103 cells $\left(2 \times 10^{7}\right)$ were washed with PBS and then resuspended with DNA in serum-free medium in a $4 \mathrm{~mm}$ path length electroporation cuvette (Fisher Scientific, Loughborough, Leicestershire, UK). Plasmid DNA, $5 \mu \mathrm{g}$ CMV- $\beta$ Gal, $5 \mu \mathrm{g}$ GR construct (if required) and $10 \mu \mathrm{g}$ reporter was added and the cells were electroporated with $200 \mathrm{~V}$ at $1500 \mu \mathrm{F}$ capacitance (Electropore 2000; SEDD, Liège, Belgium). The transfected pools of cells were divided into treatment and control groups to normalise for transfection efficiency between groups, as previously described (Ray et al. 1994, 1996, 1999, Donn et al. 2002, Huynh et al. 2002, Stevens et al. 2003a,b). Cells were harvested after $24 \mathrm{~h}$ and were lysed in $25 \mathrm{mM}$ Tris phosphate (pH 7.8), $10 \mathrm{mM}$ magnesium chloride, $0.1 \mathrm{mg} / \mathrm{ml} \mathrm{BSA}, 15 \%$ glycerol, $1 \%$ Triton X-100 and $1 \mathrm{mM}$ EDTA. Luciferase activity in $100 \mu \mathrm{l}$ of sample was measured in a Berhold Lumat LB9501 luminometer (Berhold, Pforgheim, Germany) in the presence of $0.8 \mathrm{mM}$ ATP and $0.3 \mathrm{mM}$ D-luciferin. Cells were cotransfected with $\mathrm{CMV}-\beta \mathrm{G}$ al to control for transfection efficiency as previously described (Ray et al. 1999). Results are expressed as relative light units corrected for concurrent galactosidase activity.

A549 cells $\left(5 \times 10^{5}\right)$ were transfected using lipofectamine 2000 (Invitrogen). For each transfection, $60 \mu \mathrm{l}$ lipofectamine 2000 was added to $1.5 \mathrm{ml}$ serum-free DMEM and incubated at room temperature for $5 \mathrm{~min}$. The mix was then added to a further $1.5 \mathrm{ml}$ aliquot of DMEM with the plasmid DNA. Routinely this contained $5 \mu \mathrm{g}$ reporter plasmid, $1 \mu \mathrm{g}$ CMV-renilla and, for the NCoR overexpression studies, either $10 \mu \mathrm{g}$ empty pcDNA3 or the pcDNA3-NCoR. For the NCoR over-expression studies, pools of transfected cells were divided into five aliquots for treatment with $0,1,10,100$ or $1000 \mathrm{nM}$ dexamethasone. Therefore all comparisons were made in terms of fold induction over the $0 \mathrm{nM}$ dexamethasone control within a pool of cells from the same transfection. Experiments were performed in multiples of three or four on three separate occasions with similar results.

\section{Mammalian two-hybrid analysis}

COR L103 cells were transfected via electroporation as described above, and the COS 7 cells transfected as for the 
A549 cells. COR L103 cells $\left(2 \times 10^{7}\right)$ were transfected with a 1:1:1 ratio of $5 \mu \mathrm{g}$ pG5-luc, $5 \mu \mathrm{g}$ pBIND-SRC1 and $5 \mu \mathrm{g}$ pACT-GR, or VP16/SXR. Following transfection, cells were divided into treatment and control wells, so that all comparisons were made between cells from the same transfection so eliminating transfection efficiency concerns. COS 7 cells $\left(1 \times 10^{6}\right)$ were transfected with a 1:1:1 ratio of $1 \mu \mathrm{g}$ pG5-luc, $1 \mu \mathrm{g}$ pBIND-SRC1 and $1 \mu \mathrm{g}$ pACT-GR, or VP16/SXR. Again cells were divided following transfection into treatment and control groups. Importantly, the pBIND-SRC1, which contains only a fragment of the full-length co-activator, did not activate the reporter gene alone, and neither did the pACT-GR (data not shown). After $24 \mathrm{~h}$ in the presence or absence of either $100 \mathrm{nM}$ dexamethasone or $10 \mathrm{mM}$ rifampicin (Sigma), cells were lysed in passive lysis buffer (Promega). Firefly luciferase activity in $100 \mu \mathrm{l}$ of sample was measured using the dual-luciferase assay system (Promega) as per the manufacturer's instructions. To control for transfection efficiency between transfections, a constitutively expressed renilla luciferase gene is located within the pBIND vector of the Checkmate Mammalian two-hybrid system. Renilla luciferase activity in $50 \mu \mathrm{l}$ of sample was measured, again using the dual-luciferase assay system as per the manufacturer's instructions. Results are expressed as fold induction of firefly luciferase activity after correction using the concurrent renilla luciferase activity (Stevens et al. 2003b).

\section{Western blotting analysis}

Exponentially growing cells were harvested, and lysed in RIPA buffer (100 mM Tris, pH 7•4, $150 \mathrm{mmol} \mathrm{NaCl,} \%$ NP-40, 2.5\% sodium deoxycholate and $1 \mathrm{mM}$ EDTA) with complete protease inhibitor (Roche). Protein content of the lysates was determined by using the BioRad Bradford reagent (BioRad). Three lysates were prepared on cells from different passages, and were all analysed for the presence of all the proteins below. Cell lysates $(30 \mu \mathrm{g}$ protein) were separated by SDS-PAGE and analysed with one of the following primary antibodies: anti-GR (1:500 dilution) (GR-M20; Santa Cruz Biotechnology, Santa Cruz, CA, USA); anti-SRC1 (1:100 dilution) (C-20; Santa Cruz Biotechnology); anti-NCoR (1:100 dilution) (N-20; Santa Cruz Biotechnology); anti-amplified in breast cancer-1 (AIB-1 or steroid receptor coactivator 3) (1:250 dilution) (BD Biosciences, San Diego, CA, USA) or anti-transcriptional intermediary factor 2 (TIF-2 or steroid receptor coactivator 2) (1:250 dilution) (BD Biosciences). Blocking and incubations with antibody were performed in PBS containing 5\% milk, with the exception of the anti-SRC1 and anti-NCoR antibodies, which involved blocking first in PBS containing 5\% normal rabbit serum (NRS), with antibody incubations performed in PBS containing 1\% NRS. Membranes were probed with the appropriate horseradish peroxidase-conjugated secondary antibody diluted 1:1000, and treated with enhanced chemiluminescence (Amersham International plc, Amersham, Bucks, UK) followed by exposure to film.

As the A549 and COR L103 cells expressed similar concentrations of GR we used GR abundance as a control for the quality and quantity of the protein lysates. All transfers were checked by briefly staining in Ponceau S (Sigma) before proceeding to immunoblotting. The GR antibody (M20) consistently revealed two prominent nonspecific bands, which were also used as a loading and transfer control. The specificity of the $98 \mathrm{kDa}$ band was confirmed by immunoadsorption studies. The primary antibody was incubated with a fivefold excess, by weight, of the immunogenic peptide overnight at $4{ }^{\circ} \mathrm{C}$ in PBS, then used as above to probe a membrane, divided after transfer, containing A549 protein lysates (Fig. 1C).

\section{Statistics}

Statistical analyses were by Student's $t$-test or ANOVA followed by Bonferroni $t$-test for multiple comparisons. $P<0 \cdot 05$ was considered significant.

\section{Results}

GR expression and glucocorticoid signalling in COR L103 cells

We have previously shown the $K_{\mathrm{d}}$ for the GR binding to dexamethasone to be $5 \mathrm{nM}$ in COR L103 cells (Clark et al. 1990). We therefore used a saturating concentration of dexamethasone $(100 \mathrm{nM})$ in these studies. Dexamethasone, acting at the endogenous GR, was unable to induce expression of the TAT3 reporter gene (Fig. 1A) in COR L103 cells. In contrast, the reporter gene was activated efficiently in the control, GR-positive, A549 human lung cell line (Fig. 1A). Analysis of expression of endogenous GR revealed similar concentrations of GR in the two cell lines (Fig. 1B), despite their clear differences in glucocorticoid responsiveness.

\section{GR transactivation domain function in COR L103 cells}

When a wild-type GR was transfected into the COR L103 cells, the TAT3-luc reporter gene was efficiently transactivated in the presence of dexamethasone (Fig. 2A). This confirmed that the transfected GR is expressed in the cells, that dexamethasone gains access to the receptor and that the receptor is capable of nuclear translocation and binding to DNA. Deletion of the GR C-terminal $(\triangle \mathrm{AF}-2)$, including the ligand binding and $\mathrm{AF}-2$ transactivation domains, showed a significant increase in activity compared with full-length receptor in the presence of dexamethasone (Fig. 2A); however, the relative increase in activity was less because the $\triangle \mathrm{AF}-2$ had constitutive 

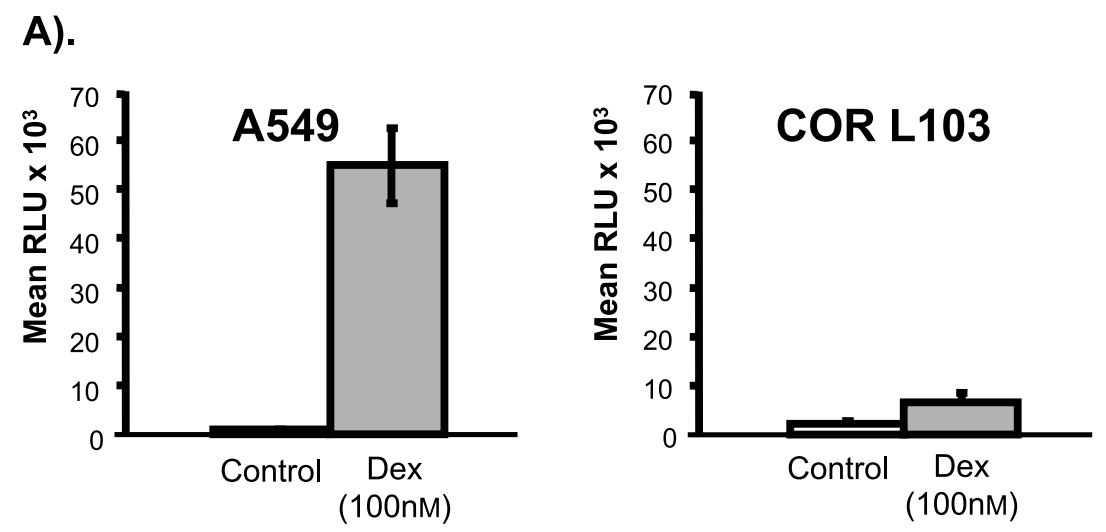

B).

A549 COR L103

98KDa

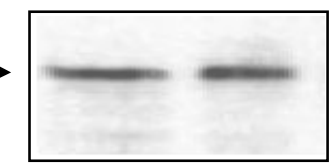

C).

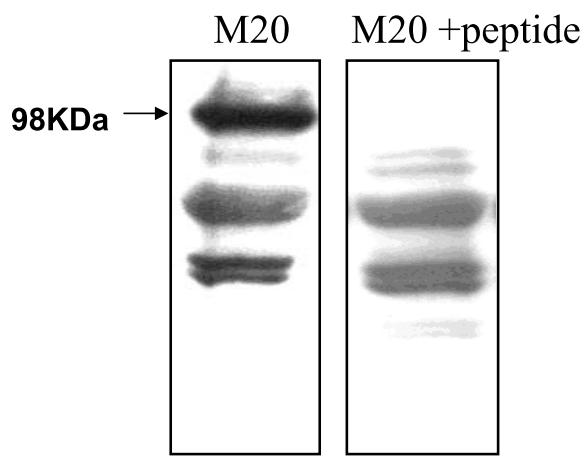

Figure 1 Glucocorticoid regulation of the TAT3-luc reporter gene in COR L103 cells. (A) COR L103 and A549 cells were transfected with the TAT3-luc reporter gene. Cells were incubated with $100 \mathrm{nM}$ dexamethasone (Dex) for $24 \mathrm{~h}$ before harvest and luciferase assay. Data are presented as mean relative light units (RLU) \pm S.D., $n=3$ individual experiments. (B) Western blot analysis with antibodies specific to GR (M20). Lysates were prepared from both A549 and COR L103 cells, and $30 \mu \mathrm{g}$ protein was resolved on $10 \%$ SDS-PAGE gels. Proteins were transferred to PVDF membrane, before immunoblotting as described. Representative results are shown; all experiments were performed on three occasions from cells of different passage, with similar results. The identity of the $98 \mathrm{kDa}$ band as the GR was confirmed by immunoadsorption studies on lysates from A549 cells. (C) M20 indicates immunoblotting with the antibody and M20+peptide indicates the use of the immunoadsorbed antibody.

activity. Loss of the $\mathrm{N}$-terminus $(\triangle \mathrm{AF}-1)$ resulted in a reduction in receptor function to $18 \cdot 3 \pm 6 \cdot 4 \%$ compared with wild-type receptor (Fig. 2B).

\section{Recruitment of SRC1 to the GR C-terminal in COR L103 cells}

Nuclear receptor sensitivity is dependent on both receptor expression and the relative expression of co-activator molecules. Protein expression of SRC family co-activators was examined using immunoblotting in COR L103, and compared with the GR-positive cell line, A549. COR L103 cells are resistant to glucocorticoids despite expressing the GR (Fig. 1), and similar concentrations of the SRC family co-activators SRC1 and TIF-2 (SRC2), and rather more AIB-1 (SRC3) compared with A549 cells (Fig. 3A). As the GR C-terminal AF-2 domain is known to recruit SRC family members in response to ligand 


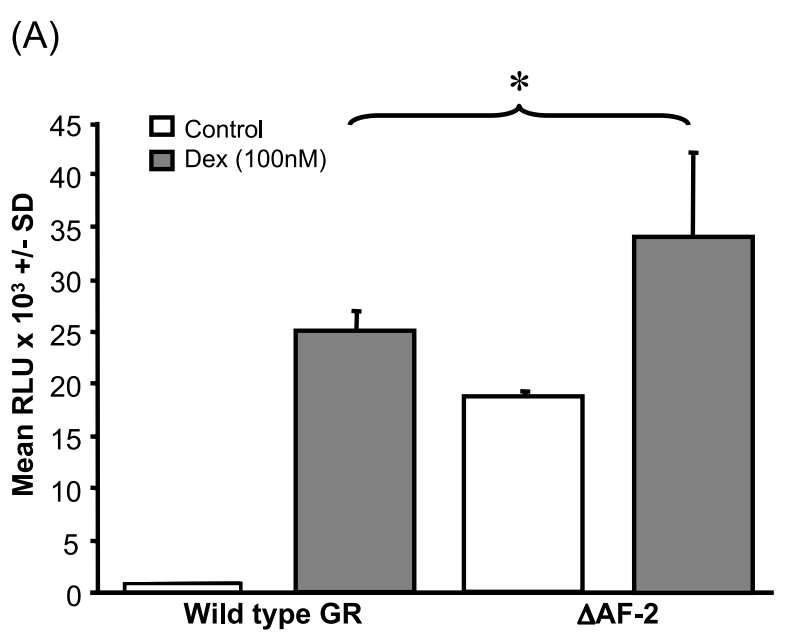

(B)

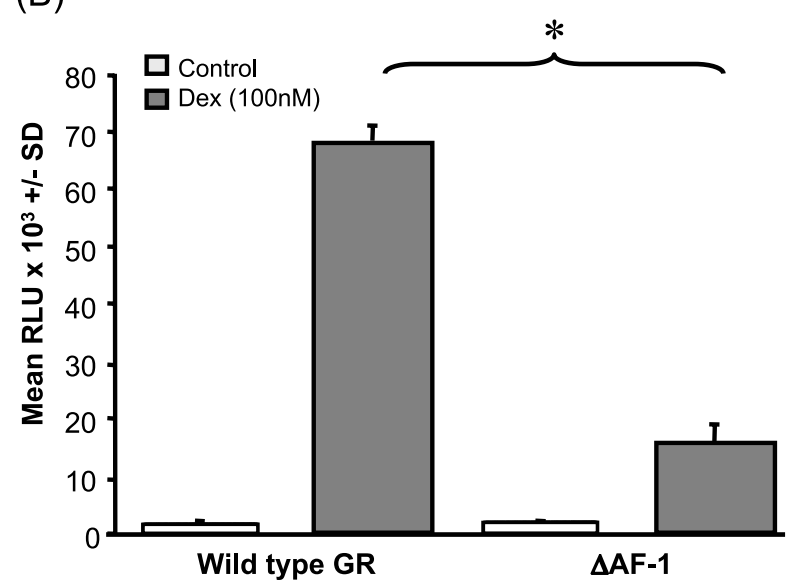

Figure 2 Activity of p6 RGR N525 and p6 RGR 407C on TAT3-luc. COR L103 cells were co-transfected with either wild-type GR or (A) p6 RGR N525 ( $\Delta \mathrm{AF}-2)$ or (B) p6 RGR 407C $(\triangle \mathrm{AF}-1)$ together with TAT3-luc. Cells were treated in the presence or absence of dexamethasone (Dex; $100 \mathrm{nM}$ ) for $24 \mathrm{~h}$. Data are presented as mean relative light units $(R L U) \pm$ S.D., $n=3$ individual experiments. ${ }^{*} P<0 \cdot 05$.

binding and as these co-activators mediate transactivation, a mammalian two-hybrid assay was used to measure interaction between the GR C-terminal and the SRC1 nuclear receptor interacting domain as described previously (Fig. 3B) (Stevens et al. 2003b). Importantly, the pACT-GR had no effect on the reporter gene with or without dexamethasone, in the absence of PBIND-SRC1. COR L103 cells showed a much-reduced interaction of GR with SRC1 in the presence of dexamethasone, in contrast to the SXR/SRC1 interaction (Fig. 3). By comparison, there was a similar level of interaction between SRC1/GR and SRC1/SXR seen in COS 7 cells (Fig. 3C and D). This suggests a GR-specific defect of recruitment in COR L103 cells.
Determination of the role of NCoR expression in modulating glucocorticoid sensitivity

As NCoR also acts through the GR C-terminal and carries transcriptional repressor activity, we measured its expression in COR L103 cells. By Western blotting, NCoR protein was clearly expressed at a higher level in COR L103 cell lines compared with the control A549 cells (Fig. 4). Therefore its effect on glucocorticoid sensitivity was assessed using the A549 lung cancer cell line as a model because A549 cells expressed similar concentrations of GR as the COR L103 cell line but had no detectable NCoR expression (Fig. 4A). NCoR overexpression resulted in a marked 'flattening' of the dexamethasone concentration/response curve in comparison with the vector only control, with maximal transactivation reduced by 55\% (Fig. 4B). Renilla activity, from a co-transfected CMV-renilla luciferase plasmid, did not differ under these experimental conditions, thereby excluding a non-specific effect on transcription (Fig. 4C).

\section{Discussion}

In the ectopic ACTH syndrome, including the aggressive SCLC, it is well recognised that glucocorticoid inhibition of ACTH can be defective. We have previously studied glucocorticoid effects in a panel of SCLC cell lines and found them to be markedly resistant to glucocorticoids but the mechanism underlying the resistance varies (Stewart et al. 1989, White et al. 1989, Clark et al. 1990). In one of the SCLC cell lines, COR L103, we have now shown that there is marked glucocorticoid resistance despite expression of endogenous GR. Importantly, the GR cDNA from COR L103 cells is free of deleterious mutations.

To understand the cause of glucocorticoid resistance in COR L103 cells, we used a TAT3 luciferase reporter gene. This is a simple, glucocorticoid-activated gene that has been extensively characterised (Iniguez-Lluhi et al. 1997, Soden et al. 2002). The AF-1 and AF-2 domains of GR act differently at the simple TAT3 reporter in COS 7 cells, in that deletion of AF-2 allows constitutive transactivation, while removal of AF-1 produces a C-terminal GR construct which shows ligand-dependent transactivation (Soden et al. 2002). In COR L103 cells, deletion of the AF-2 domain of the GR caused ligand-independent transactivation, as expected, but with a small induction in the presence of dexamethasone. One potential explanation is that the AF-1 domain of the C-terminally deleted GR is titrating out a factor within the cells responsible for repressing the function of endogenous GR such that the endogenous GR is then able to activate the reporter gene in the presence of dexamethasone. However, by comparison, deletion of the major transactivation domain in the N-terminal of the GR (GR $\triangle \mathrm{AF}-1)$ resulted in marked loss of transactivation with relatively little 
(A) $12 \mathrm{MW}$

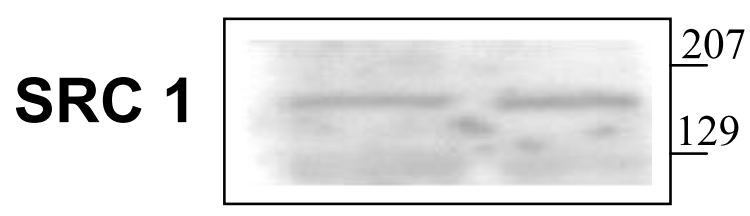

(B)

\section{Mammalian Two Hybrid Assay}
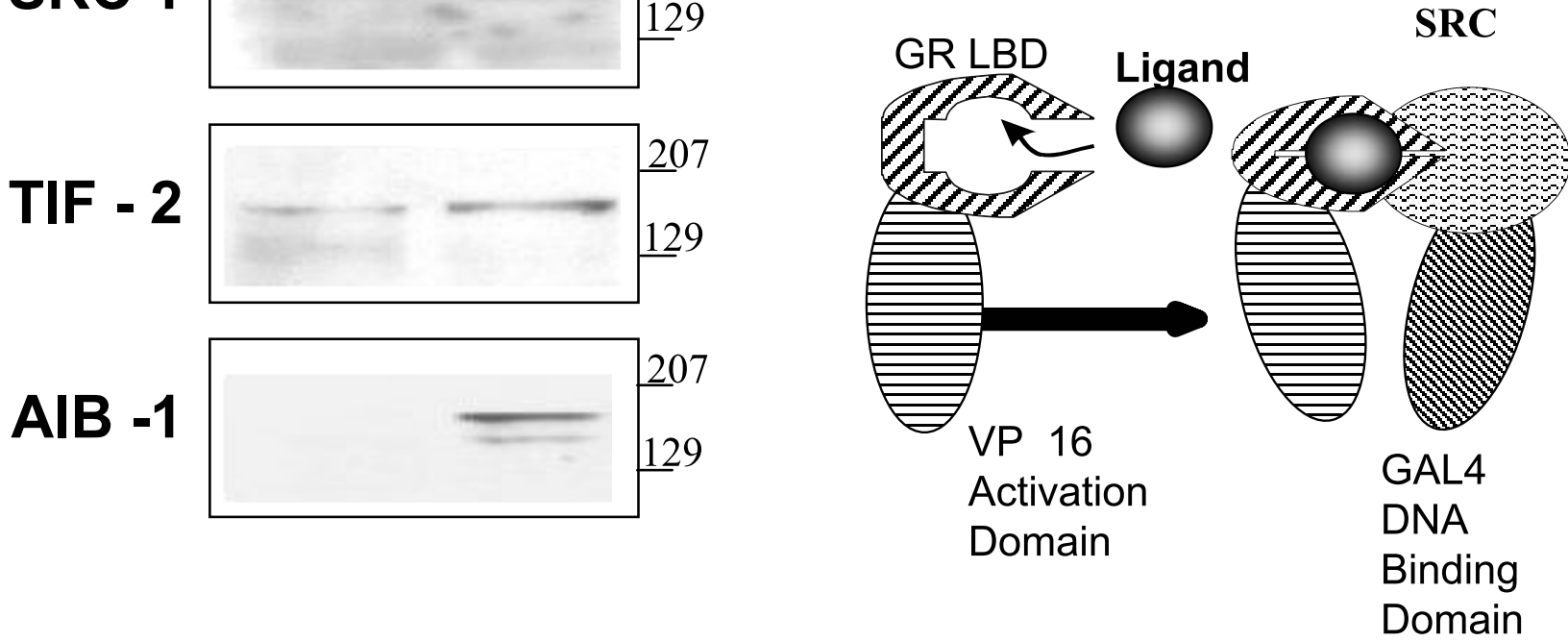

(C)
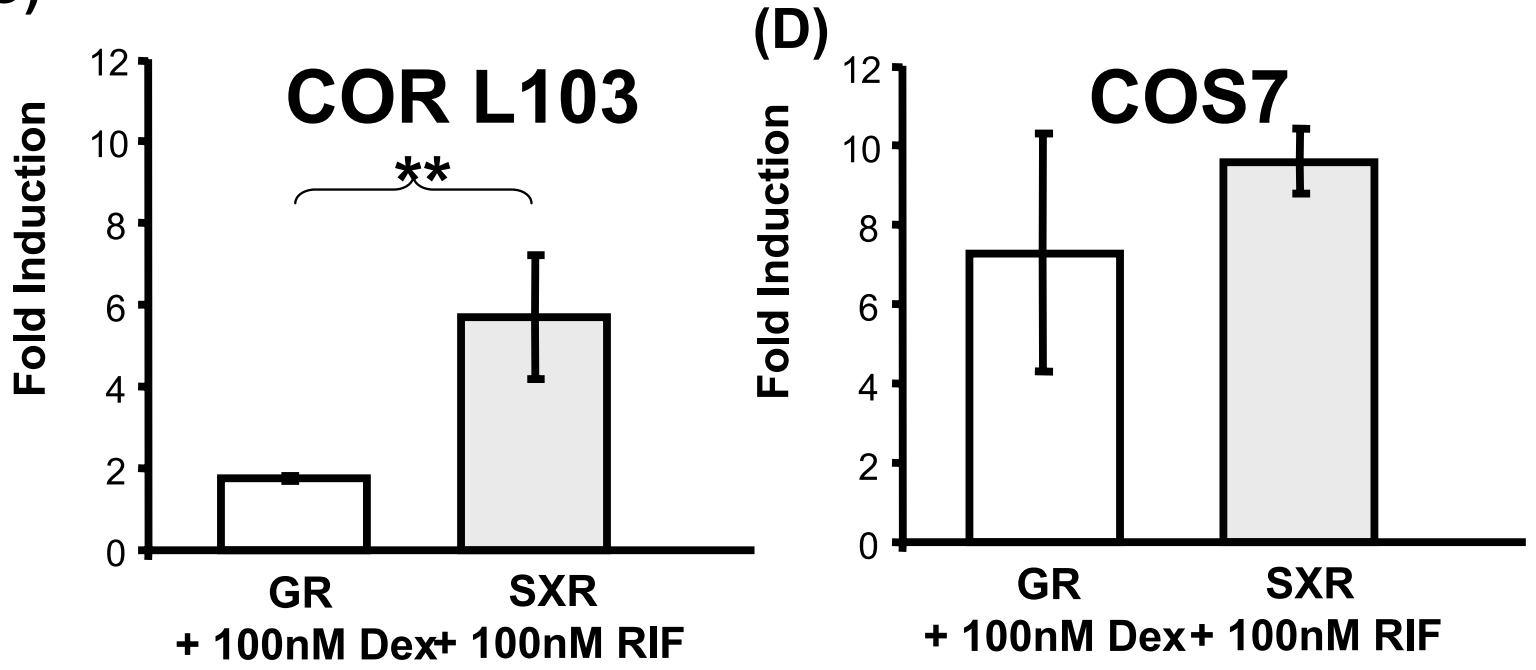

Figure 3 Co-factor recruitment to nuclear receptors in COR L103 cells. (A) Western blot analysis with antibodies specific to SRC1, TIF-2 and AIB-1. Lysates were prepared from the two cell lines, and $30 \mu \mathrm{g}$ protein was resolved on 10\% SDS-PAGE gels. Proteins were transferred to PVDF membrane, before immunoblotting as described. Representative results are shown, all experiments were performed on three occasions from cells of different passage, with similar results. Lane 1, A549 cell lysate and lane 2, COR L103 cell lysate. MW signifies molecular weight markers. (B) Model of mammalian two hybrid assay. The ligand-binding domain (LBD) of the GR is fused to the activation domain of VP16, so that if the ligand causes interaction between the GR and SRC1 transactivation of the reporter gene results. (C) Interaction between GR and SRC1 and between SXR and SRC1 in COR L103 cells. COR L103 cells were transfected with pBIND-SRC1 and either pACT-GR or VP16/SXR and the pG5-luc reporter gene (see Materials and Methods). Cells were treated with and without dexamethasone (Dex; $100 \mathrm{nM}$ ) for the GR:SRC1 interaction, or with and without rifampicin (RIF; $100 \mathrm{nM}$ ), the ligand for SXR for $24 \mathrm{~h}$. Cells were harvested and both luciferase and renilla assays performed. Corrected luciferase results were used to calculate fold induction with treatment. Data are presented as mean fold change \pm S.D., $n=3$ individual experiments. ${ }^{* *} P<0 \cdot 01$. (D) Interaction between GR and SRC1 and between SXR and SRC1 in COS 7 cells. COS 7 cells were transfected, treated and analysed as described in Materials and Methods. Results are presented as fold induction of corrected relative light units \pm S.D., $n=3$ individual experiments. There was no significant difference in the induction seen under these two conditions. 
(A)

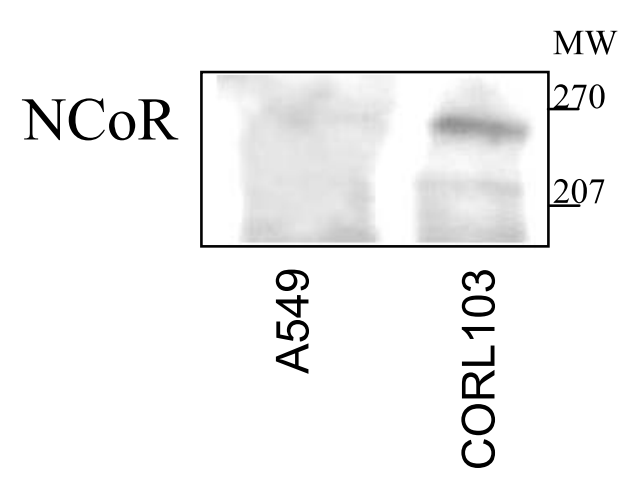

(B)

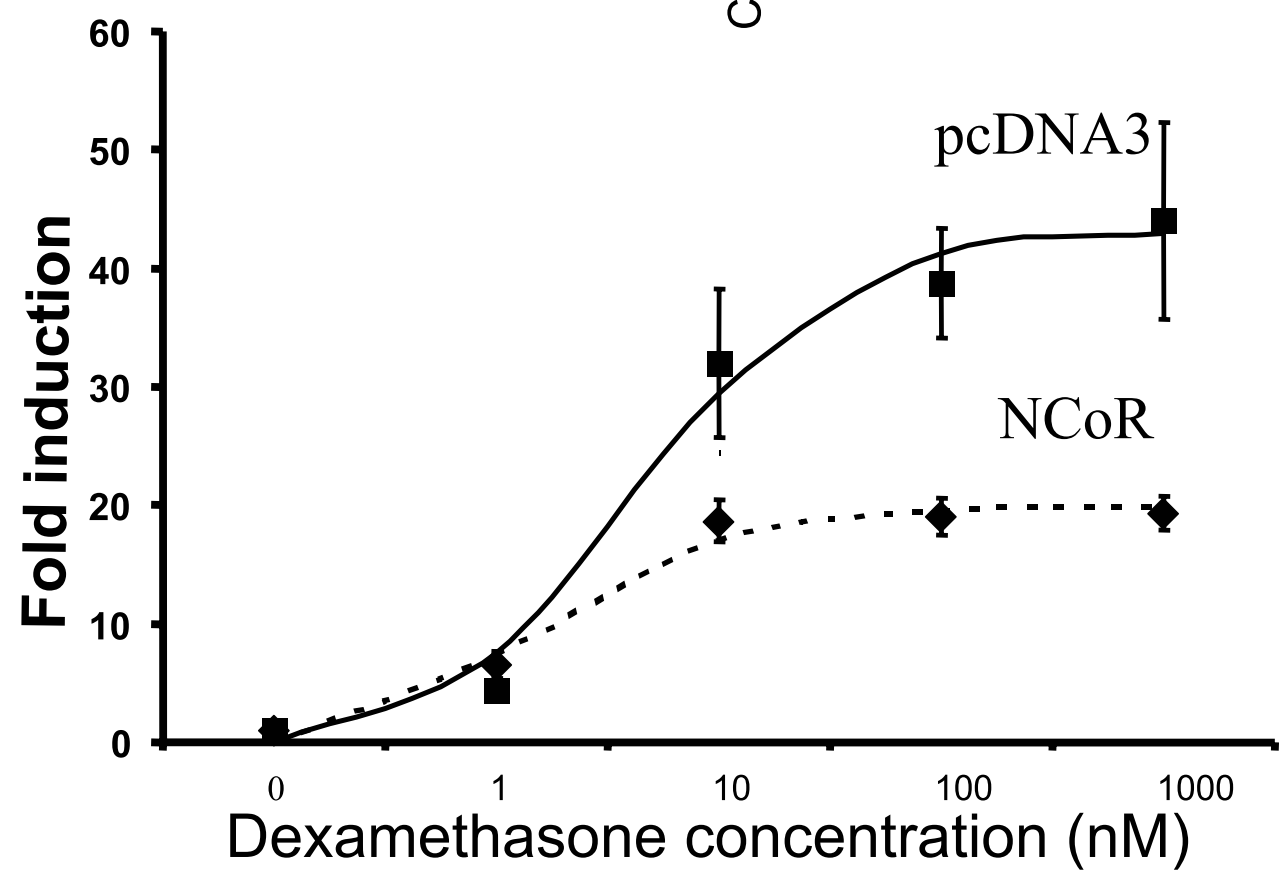

(C)

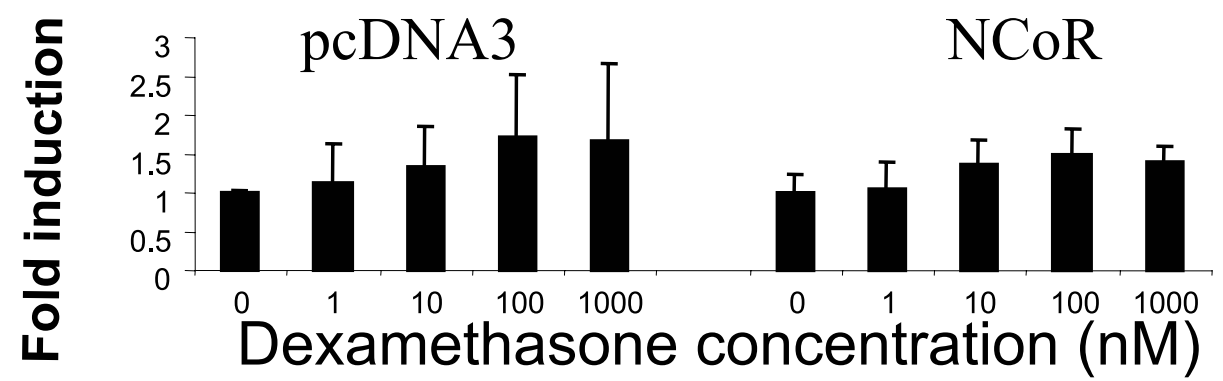

Figure 4 (A) COR L103 cells express the co-repressor NCoR. Western blot analysis with antibodies specific to NCoR. Lysates were prepared from the two cell lines, and $30 \mu \mathrm{g}$ protein was resolved on 10\% SDS-PAGE gels. Proteins were transferred to PVDF membrane, before immunoblotting as described. Representative results are shown; all experiments were performed on three occasions from cells of different passage, with similar results. Lane 1, A549 cell lysate and lane 2, COR L103 cell lysate. MW signifies molecular weight markers. (B) Modulation of glucocorticoid sensitivity by NCOR. A549 cells were transfected with either empty pcDNA3 ( $)$ or pcDNA3-NCoR ( ) and TAT3-luc. Cells were incubated with increasing doses of dexamethasone for $24 \mathrm{~h}$. Luciferase results were used to calculate fold change with treatment. Data are presented as mean fold induction \pm S.D., $n=3$ individual experiments. (C) Renilla activity is not altered by dexamethasone. The renilla activity from a CMV-renilla reporter gene co-transfected with the TAT3-luc was measured and is expressed as fold induction over the no dexamethasone control. 
ligand-dependent transactivation, in contrast to $\operatorname{COS} 7$ cells. This suggests a defect in GR C-terminal transactivation domain function in the COR L103 cells.

The GR C-terminal transactivation domain recruits co-activator molecules, including the SRC family, after agonist ligand binding (Glass et al. 1997, Szapary et al. 1999, Bledsoe et al. 2002, Stevens et al. 2003b). As the SRC proteins were expressed in COR L103 cells we measured their recruitment to the GR C-terminal in response to ligand. We have shown that the SRC1 nuclear receptor interaction domain is not recruited as efficiently to the GR C-terminal in the presence of dexamethasone in COR L103 cells compared with COS 7 cells. However, SRC1 is recruited to SXR in the presence of rifampicin in COR L103 cells. This confirmed that there is a selective, specific defect of GR C-terminal function in COR L103 cells and, as access of dexamethasone to the cells is intact, evidenced by the responses of the TAT3 reporter gene, this implies altered interactions between the GR C-terminal and other factors. The failure of SRC1 recruitment to the GR C-terminal in a mammalian two-hybrid assay performed in COR L103 cells also makes it very unlikely that simple deficiency of endogenous co-activator expression would explain the aberrant C-terminal function.

We also examined expression of the repressor protein $\mathrm{NCoR}$ which is known to interact with the C-terminal of the human GR (Schulz et al. 2002, Stevens et al. 2003b). $\mathrm{NCoR}$ is clearly expressed at a much higher level in COR L103 in comparison with A549 cells. It has previously been shown that the level of expression of co-activator and co-repressor proteins can modulate nuclear receptor function (Jackson et al. 1997, Smith et al. 1997, Lavinsky et al. 1998, Fryer et al. 2000). This is thought to provide a mechanism for subtle variation of the sensitivity of different cells and tissues to circulating hormones and vitamins. To test the implications of NCoR expression for glucocorticoid sensitivity we used A549 cells. This cell line is also derived from human lung and expresses similar concentrations of GR to COR L103. When the A549 cells were co-transfected with an NCoR expression vector there was a clear reduction in the maximal transactivation seen with dexamethasone. It may be that the overexpression of $\mathrm{NCoR}$ could compete with co-activators for binding to the C-terminal domain of the GR or, alternatively, hold the GR in a non-activating conformation. This would explain why the $\mathrm{N}$-terminal-deleted GR causes inefficient transactivation of the reporter gene.

Understanding how glucocorticoid sensitivity can be modulated both in normal cells and tissues and also as a consequence of malignant transformation has major implications for human disease (Norgaard \& Poulsen 1991). Data presented here suggest that expression of nuclear receptor co-repressor proteins such as NCoR may exert a profound effect on the function of the GR. These proteins are acting at a post-receptor level to modulate glucocor- ticoid sensitivity. It remains unclear how widespread this phenomenon is but as a mechanism identified in an SCLC cell line it may well have important implications for explaining not only aberrant peptide gene regulation (as in the ectopic ACTH syndrome) but also have relevance as a mechanism which could contribute to the malignant cell phenotype.

\section{Acknowledgements}

D W R was supported by a Glaxo SmithKline Fellowship. C E W was supported by a Biotechnology and Biological Sciences Research Council Studentship.

\section{References}

Baylin SB \& Mendelsohn G 1980 Ectopic hormone production by tumors: mechanisms involved and the biological and clinical implications. Endocrine Reviews 1 45-77.

Bledsoe RK, Montana VG, Stanley TB, Delves CJ, Apolito CJ, McKee DD, Consler TG, Parks DJ, Stewart EL, Willson TM, Lambert MH, Moore JT, Pearce KH \& Xu HE 2002 Crystal structure of the glucocorticoid receptor ligand binding domain reveals a novel mode of receptor dimerization and coactivator recognition. Cell 110 93-105.

Clark AJ, Stewart MF, Lavender PM, Farrell W, Crosby SR, Rees LH \& White A 1990 Defective glucocorticoid regulation of proopiomelanocortin gene expression and peptide secretion in a small cell lung cancer cell line. Journal of Clinical Endocrinology and Metabolism 70 485-490.

Donn R, Alourfi Z, De Benedetti F, Meazza C, Zeggini E, Lunt M, Stevens A, Shelley E, Lamb E, Ollier WER, Thomson W \& Ray DW 2002 Mutation screening of the macrophage migration inhibitory factor (MIF) gene: positive association of a functional polymorphism of MIF with juvenile idiopathic arthritis. Arthritis and Rheumatism 46 2402-2409.

Fryer CJ, Kinyamu HK, Rogatsky I, Garabedian MJ \& Archer TK 2000 Selective activation of the glucocorticoid receptor by steroid antagonists in human breast cancer and osteosarcoma cells. Journal of Biological Chemistry 275 17771-17777.

Gaitan D, Debold CR, Turney MK, Zhou P, Orth DN \& Kovacs WJ 1995 Glucocorticoid receptor structure and function in an adrenocorticotropin-secreting small cell lung cancer. Molecular Endocrinology 9 1193-1201.

Glass CK, Rose DW \& Rosenfeld MG 1997 Nuclear receptor coactivators. Current Opinion in Cell Biology 9 222-232.

Huynh TT, Ray DW, Brogan IJ, Stevens A \& White A 2002 Failure of steroid regulation of the MMTV promoter in a small cell lung cancer cell line is caused by a DNA sequence flanking the glucocorticoid response element. Journal of Endocrinology 172 295-302.

Iniguez-Lluhi JA, Lou DY \& Yamamoto KR 1997 Three amino acid substitutions selectively disrupt the activation but not the repression function of the glucocorticoid receptor $\mathrm{N}$ terminus. Journal of Biological Chemistry 272 4149-4156.

Jackson TA, Richer JK, Bain DL, Takimoto GS, Tung L \& Horwitz KB 1997 The partial agonist activity of antagonist-occupied steroid receptors is controlled by a novel hinge domain-binding coactivator L7/SPA and the corepressors N-CoR or SMRT. Molecular Endocrinology 11 693-705.

Lavinsky RM, Jepsen K, Heinzel T, Torchia J, Mullen TM, Schiff R, Del Rio AL, Ricote M, Ngo S, Gemsch J, Hilsenbeck SG, Osborne CK, Glass CK, Rosenfeld MG \& Rose DW 1998 Diverse signaling pathways modulate nuclear receptor recruitment of N-CoR and SMRT complexes. PNAS 95 2920-2925. 
Nieman LK 2001 Cushing's syndrome. In Endocrinology, edn 4, vol. 2, pp 1691-1716. Eds L DeGroot \& JL Jameson. Philadelphia: WB Saunders Company.

Norgaard P \& Poulsen HS 1991 Glucocorticoid receptors in human malignancies: a review. Annals of Oncology 2 541-557.

Odell WD 1991 Ectopic ACTH secretion: a misnomer. Endocrinology and Metabolism Clinics of North America 20 371-379.

Ray DW, Littlewood AC, Clark AJ, Davis JR \& White A 1994 Human small cell lung cancer cell lines expressing the proopiomelanocortin gene have aberrant glucocorticoid receptor function. Journal of Clinical Investigation 93 1625-1636.

Ray DW, Davis JR, White A \& Clark AJ 1996 Glucocorticoid receptor structure and function in glucocorticoid-resistant small cell lung carcinoma cells. Cancer Research 56 3276-3280.

Ray DW, Suen CS, Brass A, Soden J \& White A 1999 Structure/function of the human glucocorticoid receptor: tyrosine $\mathbf{7 3 5}$ is important for transactivation. Molecular Endocrinology 13 1855-1863.

Rivers C, Levy A, Hancock J, Lightman S \& Norman M 1999 Insertion of an amino acid in the DNA-binding domain of the glucocorticoid receptor as a result of alternative splicing. Journal of Clinical Endocrinology and Metabolism 84 4283-4286.

Schulz M, Eggert M, Baniahmad A, Dosert A, Heinzel T \& Renkawitz R 2002 RU486-induced glucocorticoid receptor agonism is controlled by the receptor $\mathrm{N}$ terminus and by corepressor binding. Journal of Biological Chemistry 277 26238-26243.

Smith CL, Nawaz Z \& O'Malley BW 1997 Coactivator and corepressor regulation of the agonist/antagonist activity of the mixed antiestrogen, 4-hydroxytamoxifen. Molecular Endocrinology 11 657-666.
Soden J, Stevens A \& Ray DW 2002 Genetic engineering of the glucocorticoid receptor by fusion with the herpes viral protein VP22 causes selective loss of transactivation. Journal of Endocrinology 172 615-625.

Stevens A, Garside H, Berry A, Waters C, White A \& Ray D 2003a Dissociation of steroid receptor coactivator 1 and nuclear receptor corepressor recruitment to the human glucocorticoid receptor by modification of the ligand-receptor interface: the role of tyrosine 735. Molecular Endocrinology 17 845-859.

Stevens A, Soden J, Brenchley PE, Cotton S \& Ray DW 2003b Haplotype analysis of the polymorphic human vascular endothelial growth factor gene promoter. Cancer Research 63 812-816.

Stewart MF, Crosby SR, Gibson S, Twentyman PR \& White A 1989 Small cell lung cancer cell lines secrete predominantly ACTH precursor peptides not ACTH. British Journal of Cancer 60 20-24.

Szapary D, Huang Y \& Simons SS Jr 1999 Opposing effects of corepressor and coactivators in determining the dose-response curve of agonists, and residual agonist activity of antagonists, for glucocorticoid receptor-regulated gene expression. Molecular Endocrinology 13 29108-29121.

Wentworth JM, Agostini M, Love J, Schwabe JW \& Chatterjee VK 2000 St John's wort, a herbal antidepressant, activates the steroid X receptor. Journal of Endocrinology 166 R11-R16.

White A, Stewart MF, Farrell WE, Crosby SR, Lavender PM, Twentyman PR, Rees LH \& Clark AJ 1989 Pro-opiomelanocortin gene expression and peptide secretion in human small-cell lung cancer cell lines. Journal of Molecular Endocrinology 3 65-70.

Received 29 July 2004

Accepted 6 August 2004 